\title{
The development of a virtual simulator for a novel design surgical tool in endoscopic endonasal transsphenoidal surgery
}

\author{
Nantida Nillahoot ${ }^{1}$, Branesh M. Pillai ${ }^{2}$, Sorayouth Chumnanvej ${ }^{3}$, Jackrit Suthakorn $^{4}$ \\ ${ }^{1,2,4}$ Center for Biomedical and Robotics Technology (BART LAB), Department of Biomedical Engineering, Faculty of \\ Engineering, Mahidol University, Salaya 73170, Thailand \\ ${ }^{3}$ Neurosurgery Division, Department of Surgery, Faculty of Medicine Ramathibodi Hospital, Mahidol University, \\ Bangkok 10400, Thailand
}

\begin{abstract}
Article Info
Keywords:

EETS

Haptic force feedback

Interchangeable surgical tool

surgical simulator

Pathway guidance

Virtual reality

\section{Article history: \\ Received Nov 6, 2020 \\ Revised Apr 4, 2021 \\ Accepted Apr 24, 2021}

ABSTRACT

Endoscopic endonasal transsphenoidal surgery (EETS) is a standard procedure to treat the pituitary adenoma, a tumor in the pituitary gland that causes malfunction of hormones. Although the method is substantially minimal invasive, the surgeon may encounter intricacies. The major challenges are narrow surgery pathway, limited working area, lack of case studies for practicing, steep learning curve owing to the intricate steps, and the tool insertion risk. To ease the neurosurgeons, this research focuses on the development and testing of the surgical simulator based on the pathway guidance and the interchangeable surgical instrument tooltip. The system was tested in human cadaver-based experiments with interchangeability in terms of function and the performance of the simulator in terms of the benefits. The experiments demonstrate the augmentation in the learning skill of the user through the simulator based on the completion time assessment and the error reduction. Furthermore, the satisfaction level of the interchangeable surgical tool, which was found using sliding switch and gripper scored $71.40 \%$, the interchangeable tooltip function, which is a novel function to participants scored $85.6 \%$ and the practical use had $77 \%$. The geometric aspect of the interchangeable tool scored lowest $(62.80 \%)$ and was found to be moderate among the neurosurgeons.
\end{abstract}

This is an open access article under the CC BY-SA license.

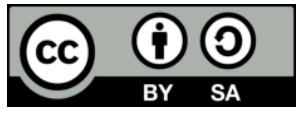

\section{Corresponding Author:}

Jackrit Suthakorn

Center for Biomedical and Robotics Technology (BART LAB)

Department of Biomedical Engineering, Faculty of Engineering, Mahidol University

999, Phuttamonthon Sai 4, Salaya, Nakorn Pathom, 73170, Thailand

Email: jackrit.sut@mahidol.ac.th

\section{INTRODUCTION}

Pituitary Adenoma is a slow-growing tumor of the pituitary gland, which is a major endocrine gland regulating necessary hormones. However, when the tumor cell grows irregularly, it might secrete excessive hormones which may result in a number of hormonal diseases and symptoms such as headache and vision loss. Basically, there are three methods available to treat the tumors: drug therapy, radiotherapy, and surgery. Regarding surgery, a technique based on natural orifices transluminal endoscopic surgery (NOTES) [1] known as endonasal endoscopic transsphenoidal surgery (EETS) [2], which accesses tumor through the sphenoid sinus from the nasal cavity, has gained considerable recognition. The EET surgical procedure [3] is initiated by the insertion of the sinus surgical tool along with an endoscope through the nasal cavity followed by the removal of the adenoma. 
EETS technique has gained popularity mainly because of the reduced recovery time, no scars, and considerably low side effects [4]-[6]. Despite these advantages, the procedure is challenged by factors such as narrow surgical pathway [7] and limited working area. Therefore, it requires the expertise of a highlyskilled surgeon [8] in order to prevent the carotid artery, cavernous sinus, and optic nerve from any harm. These factors along with the scarcity of relevant cases hinder the training for the EETS procedure. Moreover, there is also a possibility of inexperienced surgeons conducting the surgery along the wrong pathway. As there is a lack of a specific tool for EETS, it compels the surgeon to change the tools frequently during the procedure resulting in numerous tool shifts. Based on the problems, this research focuses on a system comprising of a surgical simulator based on pathway guidance and a surgical tool with an interchangeable tooltip.

To develop the novel surgical tool for EETS, the conventional tool in EETS has been taken into an account. Due to the narrow workspace [9], all the sinus surgical tools are tiny, long cylindrical, and lightweight, intended mainly for scarping and cutting the hindering bone or tissue. On the other hand, the reduction of the number of tool shifts can be achieved through the implementation of a single-port surgical tool. Compared to the Laparoscopic surgery [10]-[12], the single-port surgery relies on one incision for inserting multiple surgical instruments through the trocar-like tool [13], offering less invasiveness. For instance, the SILS ${ }^{\mathrm{TM}}$ Port is a multiple access port made up of an elastic polymer allowing flexible movement. In the direct drive system, the device is an articulated tube-shaped, attached with an endoscope and consists of a hole to insert another instrument [14]. From the concept of the single-port surgical tools, an interchangeable surgical tool was designed for EETS which is targeted at mitigating the aforementioned problems as well as reducing the invasiveness.

In order to create a realistic scenario for the surgeon, the physical properties and the environment are simulated in the simulators by creating artificial sensory experiences through visual as well as touch sensation during the training [15]. Some of the surgical simulators adhere to similar functions are virtual training system in laparoscopic surgery (Labsim), orthopedic training (MAKO RIO), a training system for Endoscopic pituitary surgery [15], [16], Otolaryngology [17], and Nephrectomy simulator [18]. Most of these researches and products ponder upon the development of a realistic environment and material properties of tissue and bone, vision, and color of each organ. However, this research gravitates towards the aspect of pathway guidance. The working methodology begins with creating the pathway guidance simulator for evaluating the conceptual function of the novel surgical tool. Generally, the simulators can be separated into three parts: anatomical model, graphical software [18], and a haptic device. Image reconstruction software plays an important role in the process and calculates the virtual world based on the mathematical model to acquire the anatomical 3D model by applying medical imaging [19]. Many of the software has been launched such as itk-SNAP, Invesalius, 3D Slicer, and Osiris [19]. After comparing the performances, a 3D slicer has been chosen for creating the model using semi-automatic segmentation [20], to obtain the model of pituitary tumor and nasal structure [21], [22], which is exported into mesh model [23].

Within graphical software, the game engine [24] has been used for creating a realistically visual environment to display and sensation to interact with the user [25]. There is a glut of open source software such as Unity, PhysX, Unreal engine, and id tech [26] that can be used, for integrating medical data and also the physical and material properties of human anatomy. Unity is the most suitable platform due to the compatibility with the haptic device, computer-aided design (CAD) supporting, rendering performance, and an extensive parameter option for physical properties [27]. The final component within the simulator is the haptic device which is a peripheral device that provides a physical contact interface between the computer and the user [28]. In a surgical simulator, the surgeon can sense the imitated touch like in real surgery from the device which acts as the surgical tool. Occasionally, the device's handle is modified to attach the surgical tools to facilitate more specific practices [29]. In this research, the haptic device stylus is modified with the multi-surgic-tip tool, and the haptic interact model is developed for testing the conceptual design of the interchangeable tooltip surgical tool with participants who are experienced neurosurgeons. The interchangeable tooltip surgical tool is developed to overcome the tool's shifting problem.

\section{RESEARCH METHOD}

\subsection{Overview}

In this research, there are three main aspects, comprising of Haptic-VR surgical simulator for EETS pathway guidance as shown in Figure 1(a), interchangeable tooltip surgical tool, and the multi-surgic-tip tool as shown in Figure 1(b). The interchangeable tooltip surgical tool is a novel design surgical tool for EETS made to overcome the problem of numerous tool shifting during the procedure. To evaluate the conceptual design of the novel surgical tool, Haptic-VR surgical simulator for EETS pathway guidance is developed. The simulator can also be used for the practice of the motor hand control movement of the users by simulating the surgical environment. The multi-surgic-tip tool is a modified haptic stylus that attaches with 
the handle of the interchangeable tooltip surgical tool. The tool provides the function and the appearance in such a way that both the evaluation and simulation can be performed.

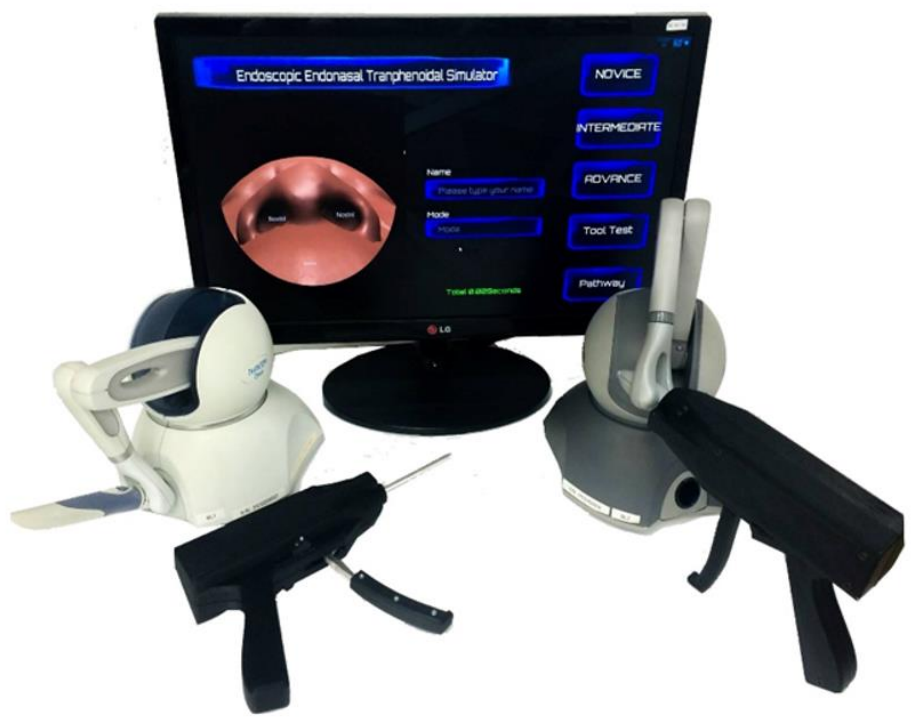

(a)

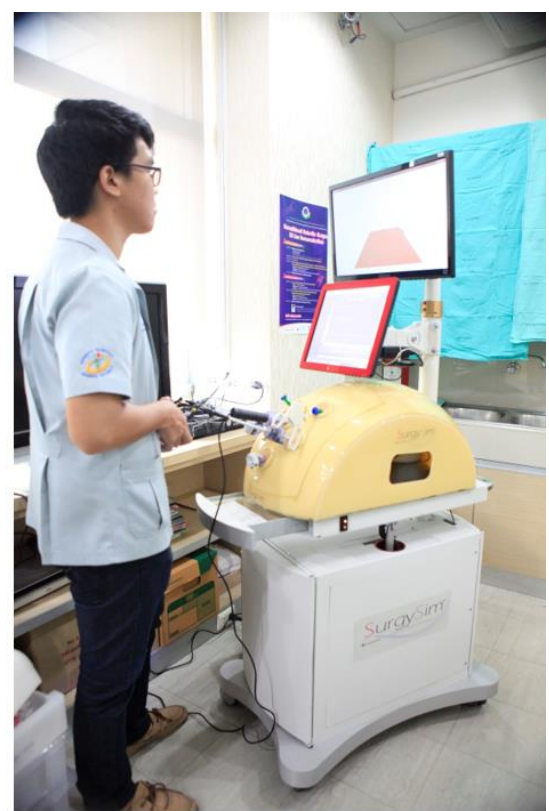

(b)

Figure 1. BART LAB Haptics-VR surgical training simulation system, (a) EETS pathway guidance system, (b) SurgiSim-abdominal surgical training system

\subsection{The interchangeable tooltip surgical tool for EETS}

The design of the interchangeable surgical tool was performed in three steps. The first stage was the review of the design of the sinus surgical tool model and haptic device through the evaluation and assessment in terms of dimension, function, and mechanism. The major aspects of considerations were suitability with the procedure of EETS, the convenience of usage, and the functionalities of the surgical tooltip. The second step was the construction of the CAD model of the tool to conduct the motion study in Solidworks Motion Studies. The final stage was the generation of the mesh model of the tool and introducing the model within the simulator for the integration with the haptic devices. After the completion of these three stages, the Phantom Omni stylus was modified to evaluate the design with the participants who were some experienced surgeons in EETS. The interchangeable tooltip surgical instrument serves two functions. The first function targets to ease the switching of tooltips in a single instrument. It eschews the tedious insertion process through the sophisticated nasal pathway. Moreover, the overall procedure can be performed through a onehanded operation, which ensures convenient usage for the neurosurgeon. The second function is the punching mechanism that is activated specifically for the cutting tools.

The design of an interchangeable tooltip instrument involves three mechanisms to facilitate the two functions as mentioned above. First, the tooltip extension mechanism, which is for the stretching and gathering of the tooltip during the usage and non-usage phases respectively and applied by a mounted scissor mechanism into a slide button. As illustrated in Figure 2, the scissor mechanism also provides a proportional stroke length during the sliding movement. Hence, this mechanism can maintain the dimensional integrity of the instrument to fit in a limited space in the human's nasal cavity while making it possible for the switching of the tooltip by one-handed use.

Secondly, the tooltip switching mechanism is used to change the two tooltips: Kerrison Rongeur and Ring Curettes. This mechanism consists mainly of retracting boxes and retract rod. The retract box is used for locking and unlocking the tool's position. The tooltip extension mechanism and the tooltip switching mechanism coordinate and collaborate. Lastly, the punching mechanism in this case uses Kerrison Rongeur, which performs like scissors for the cutting operation, and are also available in the conventional surgical tools.

\subsection{The EETS pathway guidance surgical simulator}

The virtual reality-based EETS simulator consists of three main components. The first component is the combination of the 3D data obtained from the real patients and the cadavers. These data include the 3D 
mesh model of the nasal cavity acquired from the medical image data (CT scan of the real patients) and the pathway model obtained from the optical tracking sensor data (EETS pathway of the Cadavers). This step demands the image reconstruction software like a 3D slicer to select the area of the nasal cavity and nose skin in each medical image segmentation. Then the mesh model is imported into the CAD file to study about anatomical structure and position of the sphenoid sinus and the pituitary tumor which will be used as the environment of surgery in the simulator. For the pathway model, MATLAB is used to apply the statistical analysis data from sensors to find the average path for tool insertion.

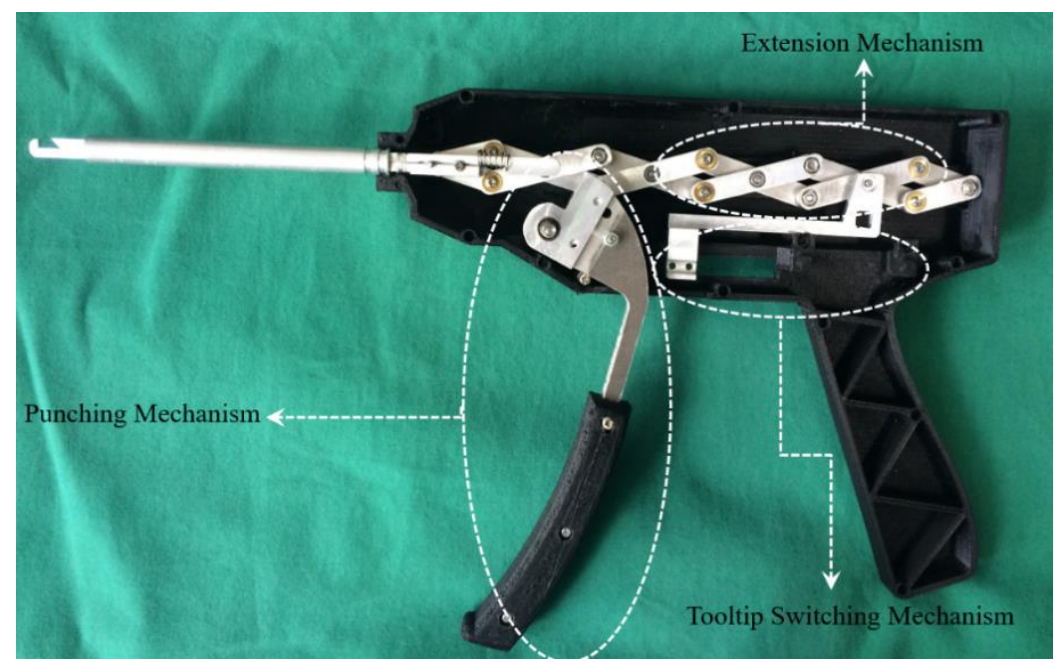

Figure 2. Design of the interchangeable tooltip surgical tool

The second component of the simulator is the haptic device which sends force feedback from the virtual world to the real world. It requires hardware called "Phantom Omni", which enables the touching perception simulation. The last component is the game engine that conducts the interaction between the user and the virtual world by sending visual sensations and touch using Unity, which can simulate the 3D model of the nasal cavity and has a plugin for working with Phantom Omni device. Besides, the software will also act as a central processing unit, collecting both the position and orientation data from the haptic device and generating the tactile information for allowing the user to feel the force-feedback sensation. The CAD model of the nasal cavity, EETS pathway, and EETS surgical tool are imported as mesh models and the stylus of Phantom Omni is modified with the novel design tool. The virtual world of EETS tool insertion along with the nasal cavity model is shown in the display monitor.

\subsection{Creating the surface model}

In this simulator, four models are used as a surface boundary for the haptic device sensation. The polygon surface model or mesh model consists of the Nasal cavity (Visualization), Nasal cavity (Haptic rendering), Pathway line, and Head model as shown in Figure 3. To create the mesh model, the image reconstruction software is required to analyze the file type, digital imaging, and communications in medicine (DICOM).

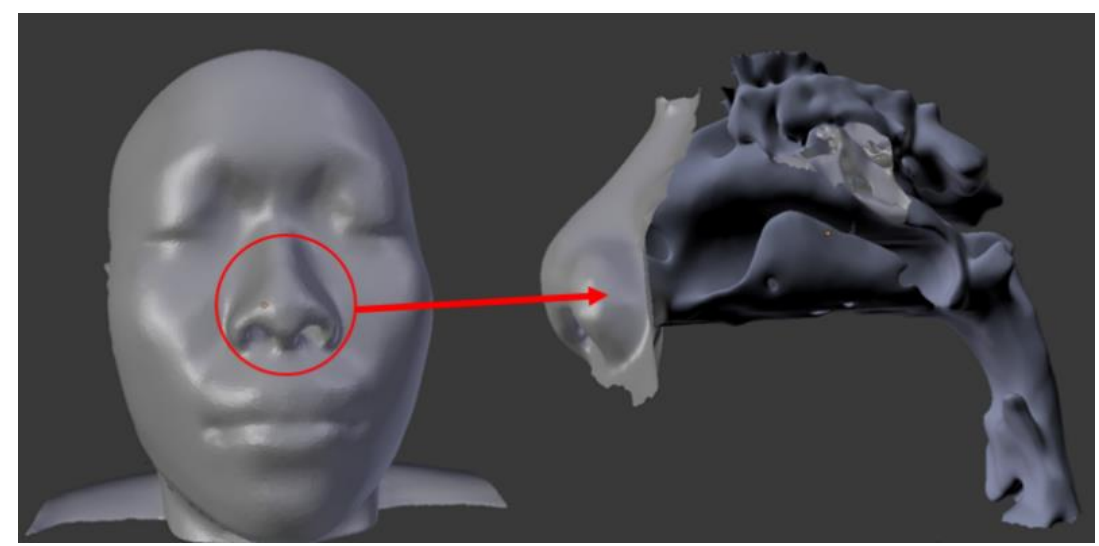

Figure 3. Polygon surface model in the simulator 
The model presented is constructed from the high-resolution computational tomography (CT) images of the human skull with a slice thickness of $1.0 \mathrm{~mm}$ in $512 \times 512$ matrix to create the surface model with "3D slicer" [25]. The model is separated into two components: a high number polygon surface, which can cause a problem to the haptic device when calculating the force feedback, and a lower polygon model. The nose model is converted by "Blender", a graphic software suitable for reducing the number of polygons while preserving the topology of the model. The experimental data of the optimum pathway for EETS from the related study that obtains the position of the tooltip movement was imported into Blender and a pathway was created. Finally, a circular surface is created along the path.

\subsection{EETS simulator}

The game engine software "Unity3D" is used to create a real-time graphical image and simulate the surgical environment. The advantages of using a game engine to create a surgical simulator are that the physics engine in the system can set the parameters such as gravity, velocity, friction, collision, and light to mimic the real world. Four surface models, as mention above, are imported into the game engine to use as material to create the virtual EETS surgery. The surgical simulator requires realistic visualization and sensations. In this system, tool action and perception interact with the user by a commercial haptic device, Phantom Omni (Sensable Technologies), with three degrees of freedom for force feedback to the user and six degrees of freedom for motion. Real-time position and rotation from the device are directed to the software using the Phantom Omni Plugin for Unity 5.0.

The main features of the EETS Simulator software for the user are endoscopic views like a Sinoscope, operating scene from a third-person view, transparent nose model to show the position of the virtual surgical tool, and the information panel to show the time elapsed including the distance from the sphenoid sinus. The user can see the anatomical structure of the nose interior on the left side of the screen. During practice, the software generates a boundary from the polygon to limit the movement of the haptic device position and releases the force feedback to the user as shown in Figure 4.

\subsection{Experimental protocol for EETS simulator}

The subject in this study comprises of neurosurgeons and biomedical engineering students. Specifically, the seven practicing neurosurgeons are proficient in EETS while the fifteen biomedical students have no prior experience in EETS. The experiment was conducted and completed with practice on 3 modes which are novice, intermediate, and advance, consecutively as shown in Figure 5.

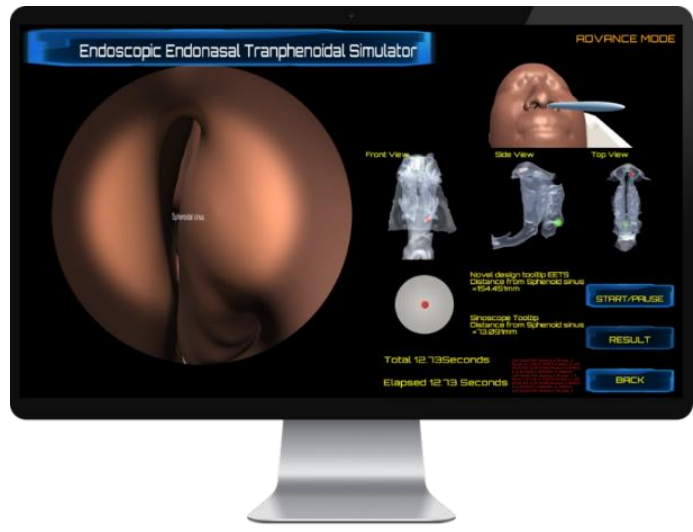

Figure 4. User interface of the EETS simulator shows the endoscopic view on the left and navigation panel on the right

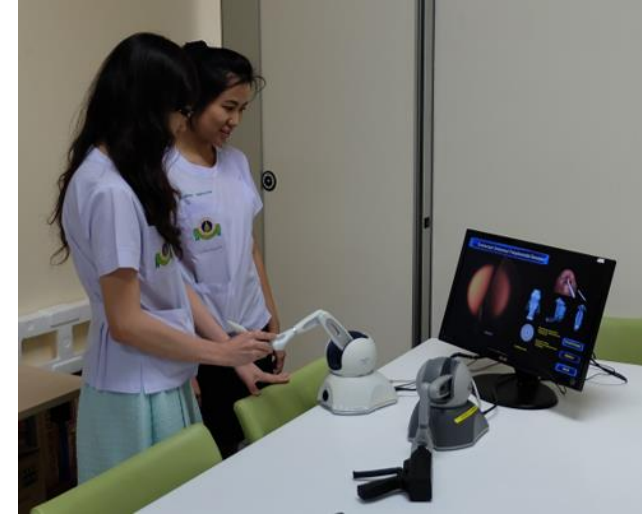

Figure 5. Testing the pathway guidance surgical simulator with the participants at Ramathibodi Hospital

The practice was performed over three trials in each mode starting from the Novice step, which is the easiest level to the most difficult advance level. The advanced level closely simulates the situation in real operations. The modes have been classified based on the level of assistance the system concedes to the user. Specifically, certain criteria as mentioned in Table 1 are the basis for categorization. As mentioned, nose boundary refers to the sensation depicting the initial nose cavity boundary. The guidance force refers to the force assistance throughout the motion while pathway boundary refers to the virtual sensation matching the pathway throughout the operation. Novice mode, which is considered the easiest, assists nose boundary, 
guidance force and pathway boundary. Similarly, intermediate mode assists the nose boundary and guidance force. The advanced mode is the most challenging as it only grants assistance for the nose boundary while further navigation is unassisted and relies entirely upon the user. The simulator system collects the data for each training by recording the time required to complete a practice, error score during the practices, and transmit all these data to store on a computer.

Table 1. Criteria for categorizing practice mode of the simulator

\begin{tabular}{cccc}
\hline & Nose Boundary & Guidance Force & Pathway Boundary \\
\hline Novice Mode & $/$ & $/$ & $/$ \\
Intermediate Mode & $/$ & $/$ & $\mathrm{X}$ \\
Advanced Mode & $/$ & $\mathrm{X}$ & $\mathrm{X}$ \\
\hline
\end{tabular}

Error score is calculated from the collision of the haptic tooltip with the cross-section path along the path boundary, which is masked as a transparent object in Unity3D. In (1) is used for the simulator error score calculation.

$$
\text { Score }=\frac{\sum_{n-1}^{c} S_{n}+S_{\max } *(T-C)}{n}
$$

where;

$\mathrm{C}=$ collided path

$\mathrm{n}=$ number of sample

$\mathrm{S}=$ radius of each cross section

$\mathrm{T}=$ total number of cross section path

Note* Out of area will concern as max distance from pathway line

\subsection{Experimental design for testing novel design}

The interchangeable tooltip surgical tool function was tested with the surgical simulator via the pathway guidance. The haptic interact model consisting of the multi-surgic-tip tool was fabricated to interface the new surgical tool for the participant and neurosurgeons as shown in Figure 6. A test was conducted to perform a comparative assessment of the performance between the interchangeable surgical tool and conventional surgical tool through the simulator. A questionnaire was used to evaluate through a survey among the neurosurgeons who had experience with the EETS procedure, focusing primarily on the changeable tooltip function. In the survey, the questions were aimed at comparing the interchangeable tooltip surgical tool use in terms of satisfaction of the tool's function with the EETS conventional tool as shown in Figure 7.

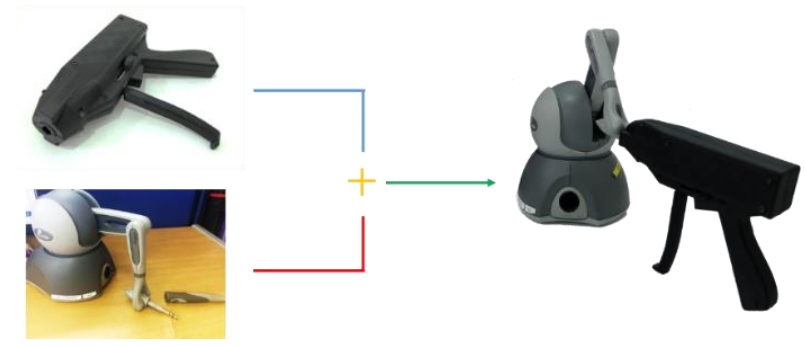

Figure 6. The haptic interact model attached with the Phantom Omni haptic device's stylus, developing into a multi-surgic-tip tool

Within the interchangeable tooltip surgical tool, there are two movements: button sliding to use tooltip on each side and the handle griping for cutting. Therefore, the model has two sliding buttons and a gripping handle. The sliding button simulates the usage of the changeable function and the gripping handle assumes the cutting function. For interfacing with the haptic device, the model is attached to the Phantom Omni haptic device's stylus. The stylus consists of two-button switches for sending the digital inputs to the simulator as shown in Figure 8.

The stylus's button operates with four communicated commands in which 2 switches are not in use, use switch A only, use switch B only, or use 2 switches. The electronic switches are applied in a multi-

The development of a virtual simulator for a novel design surgical tool in endoscopic... (Nantida Nillahoot) 
surgic-tip tool model for detecting all the movements. The micro-switches are mounted into the model to detect the slide button movement and command the simulator to display the changeable tooltip function in virtual reality. The selected tooltip would penetrate out of the virtual tool. For the handle gripping movement, the pushbutton switch is used for detecting the griping handle when the tool is cut by the user. Similar to the changeable function, the cutting would be displayed in the simulator.

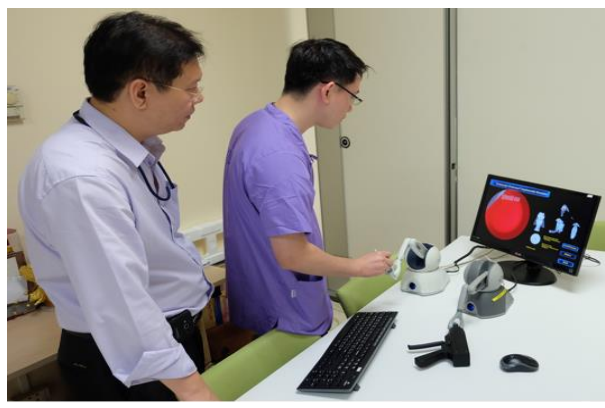

Figure 7. Testing the interchangeable tooltip surgical instrument function with an EETS simulator and multi-surgic-tip tool with participants, the neurosurgeons

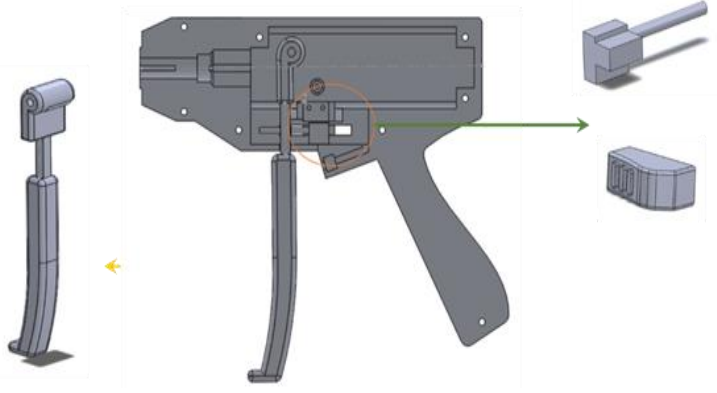

Figure 8. Components in the haptic interact model; the micro-switches for detecting sliding buttons and the pushbutton switch for detecting the handle gripping movement

\section{RESULTS AND DISCUSSION}

The total number of trials performed amounted to 198 trials, with all the subjects completing the 3 modes of the simulator. The analysis of the data gave the time to completion and the error score for each model. Using Gaussian distribution is represented in Figures 9-11. The three groups of data show the different training at the first, second, and third trials respectively. The trending of the data from the Gaussian plot shows that the standard deviation and the results are shown in Tables 2-4.

\subsection{Novice mode}

In this mode, the user can perform the fastest time compared to others due to the assistant of the pathway guidance system which consists of pulling force, pathway boundary, and nose boundary. These factors help the user to navigate and locate the area of the sphenoid sinus effectively as shown in Figure 9(a).

When a virtual surgical tooltip collides with an invisible circular surface aligning along the pathway line, the error score can be calculated by (1) as mentioned before. Error score for each trial is represented by box plot in Figure 9(b) which shows that variance and mean of error can be decreased when trainee perform until the third trial as shown in Table 2.

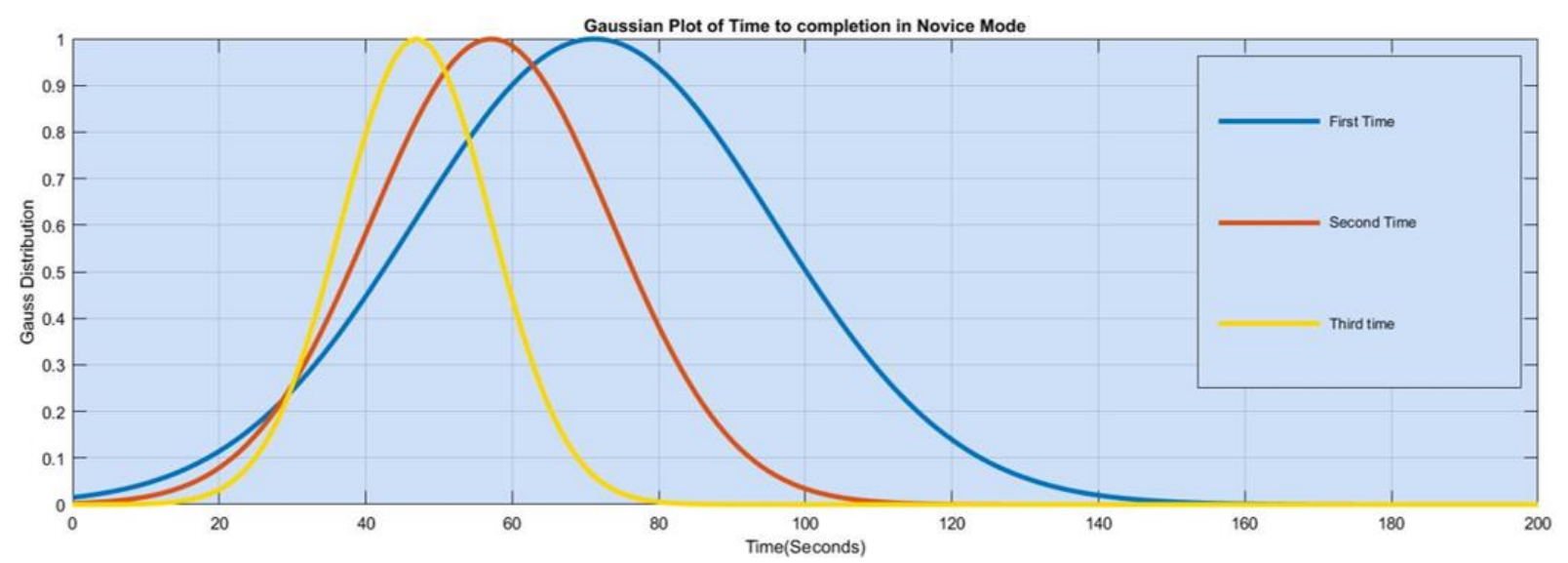

(a)

Figure 9. Novice mode, (a) Gaussian plot of time to completion 


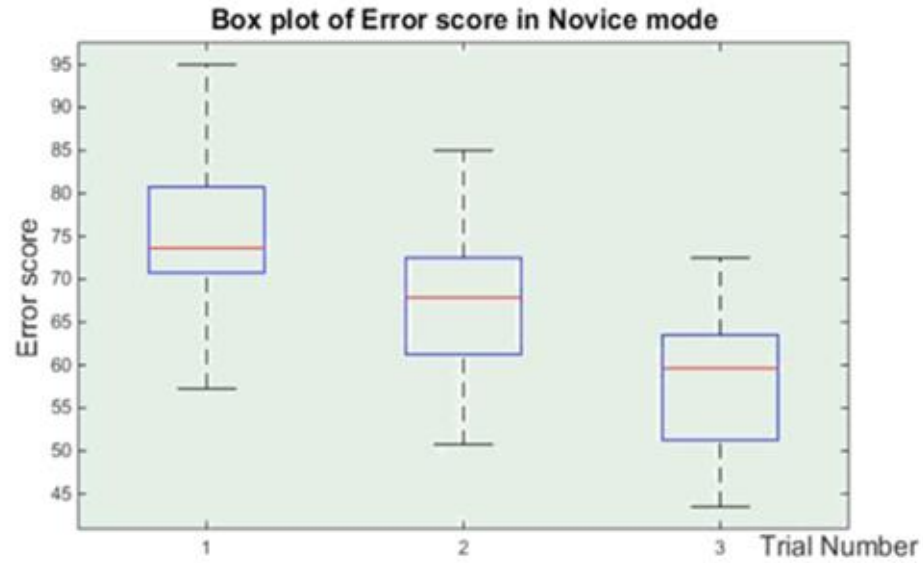

(b)

Figure 9. Novice mode, (b) Box plot of error score (continue)

\subsection{Intermediate mode}

This mode allows the user to move the surgical tool freely within the nasal cavity by removing the pathway boundary. Therefore, this mode is more difficult than Novice mode as the user can even move to the wrong locations such as the esophagus or another nasal sinus. From the graph in Figure 10(a) and Table 3, it shows that the user performs the task longer than Novice mode which is supported by the slight increase in the mean and variance value. However, the system can assist the user by giving lower time for completion.

The range of error score in Intermediate mode is more than Novice mode due to the complicated pathway after removes the pathway boundary as shown in Figure 10(b). Furthermore, the Table 3 shows that the mean and the variance value still decreased while practicing with the simulator.

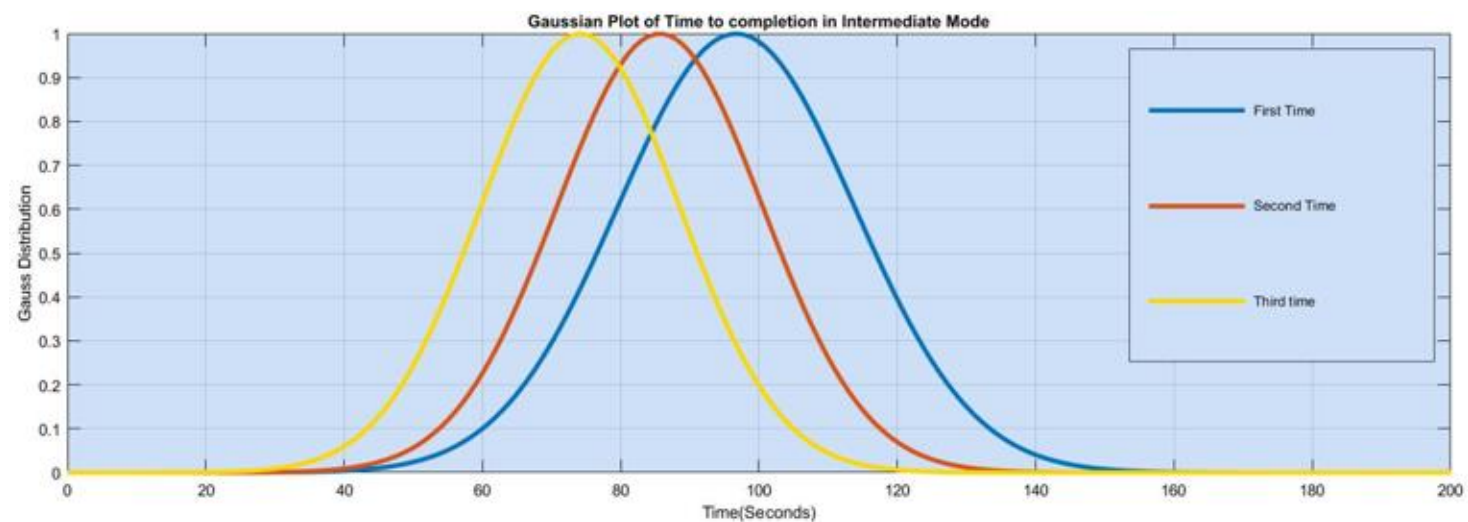

(a)

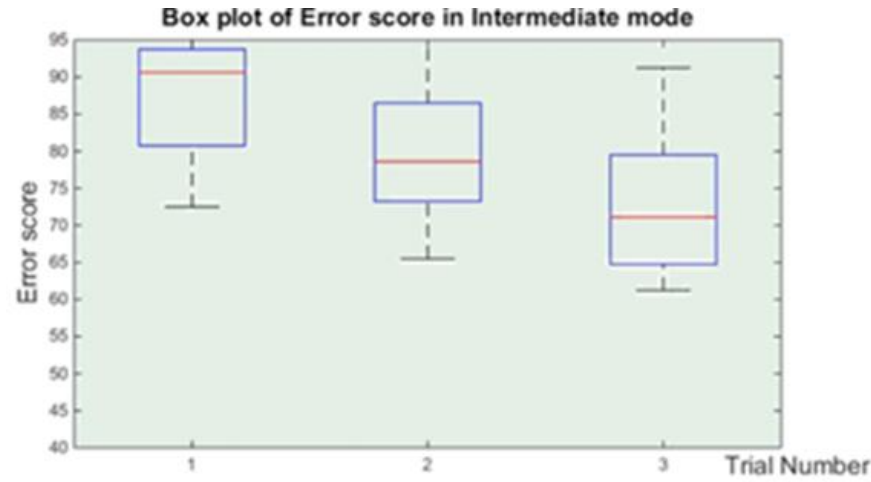

(b)

Figure 10. Intermediate mode, (a) Gaussian plot of time to completion, (b) Box plot of error score

The development of a virtual simulator for a novel design surgical tool in endoscopic... (Nantida Nillahoot) 
Table 2. Mean and variance of time to completion and error score in novice mode

\begin{tabular}{cccc}
\hline Novice mode & Trial \#1 & Trial \#2 & Trial \#3 \\
\hline \multicolumn{5}{c}{ Time to completion in Novice mode } \\
Mean & 71.18 & 57.13 & 46.92 \\
Variance & 604.25 & 271.45 & 105.13 \\
\multicolumn{5}{c}{ Error score in Novice mode } \\
Mean & 75.39 & 68.36 & 58.32 \\
Variance & 81.24 & 77.71 & 71.24 \\
\hline
\end{tabular}

Table 3. Mean and variance of time to completion

\begin{tabular}{cccc}
\multicolumn{4}{c}{ and error score in Intermediate mode } \\
\hline Intermediate mode & Trial \#1 & Trial \#2 & Trial \#3 \\
\hline \multicolumn{4}{c}{ Time to completion in Intermediate } \\
Mean & 95.68 & 85.63 & 74.13 \\
Variance & 292.41 & 221.19 & 206.50 \\
Error score in Intermediate mode & \\
Mean & 88.60 & 79.86 & 72.62 \\
Variance & 62.40 & 85.77 & 82.30 \\
\hline
\end{tabular}

\subsection{Advanced mode}

Pathway boundary and pulling force have been removed for this mode to allow the user to practice under an environment similar to that of real surgery. The result from this mode shows that the trainee spends more time according to Figure 11(a), where the base of the Gaussian plot extends more than the other modes. The mean and the variance value declined as shown in Table 4.

Error score in advance mode can be described by Figure 11(b), which shows significant variation in the size of the box plots for each trial. The difficulty of this mode made the trainee move the surgical tool out of the appropriate EETS pathway. There is some sample that trainee got the highest error which is considered as the outlier of the data. According to Table 4, the mean error score decreased, while on the other hand, the variance has increased due to the trainee becoming unaware of the complicated pathway. The main way to solve this problem is through relentless practice within the system.

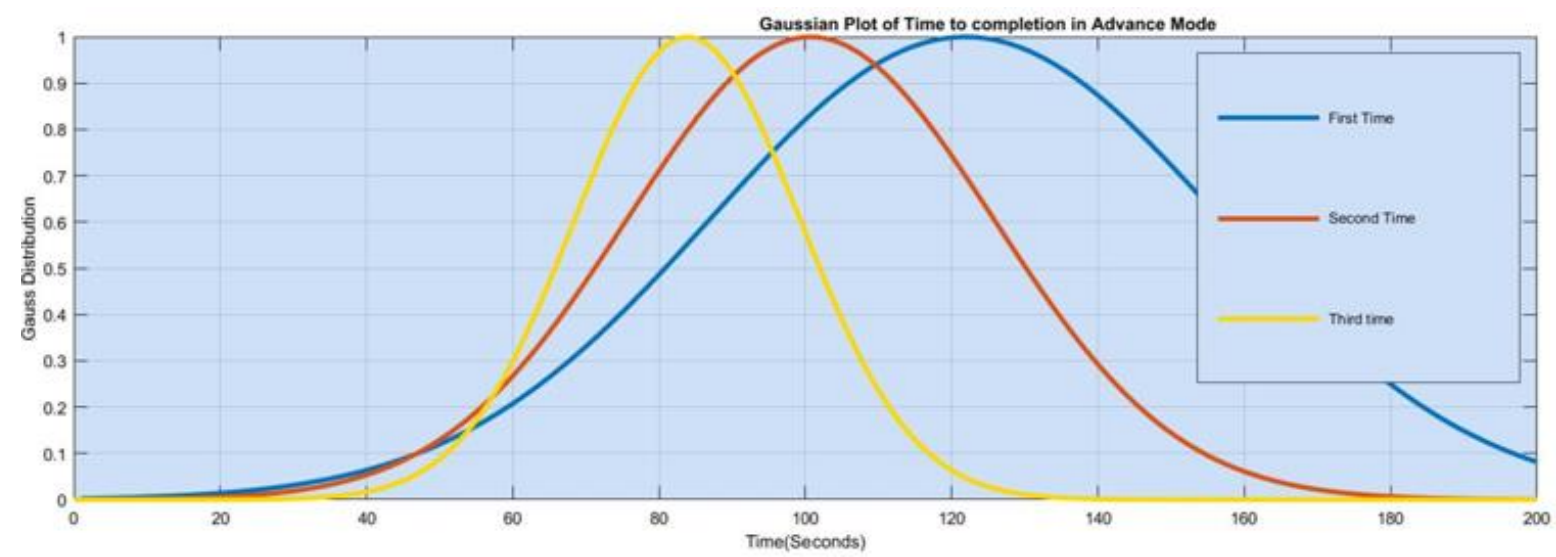

(a)

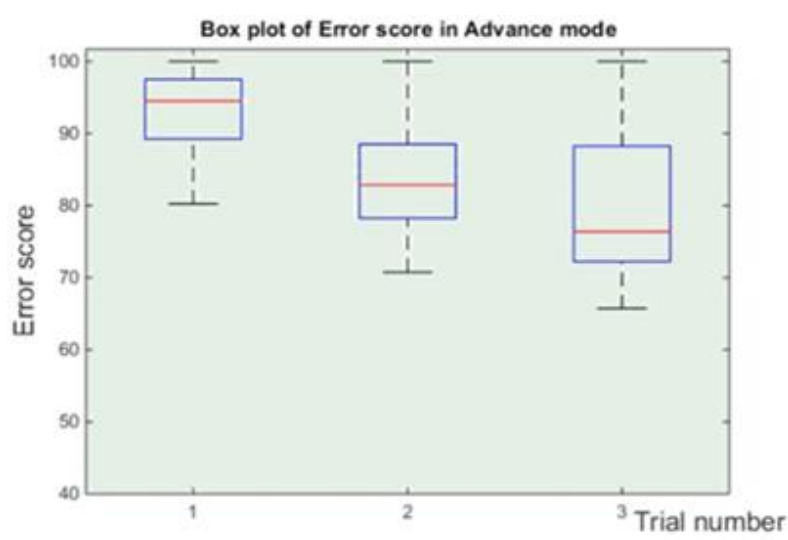

Figure 11. Advance mode, (a) Gaussian plot of time to completion, (b) Box plot of error score

\subsection{Interchangeable tooltip surgical tool}

The experimental result of the interchangeable tooltip surgical tool is presented in Table 5 from the evaluation survey with 7 neurosurgeons who were experienced in the EETS procedure. The result of the test 
pertaining to the EETs simulator and the multi-surgic-tip tool has been quantified in terms of percentage based on different criteria such as size and shape, the convenience of using the sliding switch, and gripper, interchangeable function, and ergonomics.

Table 4. Mean and variance of time to completion and error score in advance mode

\begin{tabular}{cccc}
\hline Advance mode & Trial \#1 & Trial \#2 & Trial \#3 \\
\hline \multicolumn{4}{c}{ Time to completion in Advance } \\
Mean & 120.86 & 100.61 & 83.86 \\
Variance & 1216.98 & 627.56 & 236.21 \\
Error score in Advance mode & \\
Mean & 93.44 & 83.56 & 80.01 \\
Variance & 31.06 & 85.77 & 99.12 \\
\hline
\end{tabular}

Table 5. Results from questionnaires which survey from the neurosurgeons

\begin{tabular}{lc}
\hline \multicolumn{1}{c}{ Description } & $\begin{array}{c}\text { Mean satisfaction } \\
\text { score }\end{array}$ \\
\hline $\begin{array}{l}\text { Size and shape of tool } \\
\text { Convenience of using switch and }\end{array}$ & $62.80 \%$ \\
gripper & $71.40 \%$ \\
$\begin{array}{l}\text { Interchangeable tool function } \\
\text { Practical use of design }\end{array}$ & $85.6 \%$ \\
\hline
\end{tabular}

Comparing with the conventional surgical tool from the participants' experience, most of the topics in the questionnaire had a satisfaction rate of over 70 percentages, which shows the concept design of the interchangeable tooltip tool to be acceptable in the EETS procedure. Regarding the satisfaction levels, the convenience of using sliding switch and gripper scored 71.40 percent, the interchangeable tooltip function, which is a novel function to participants scored 85.6 percent and the practical use had 77 percent. However, the lowest score was received by the size and shape aspect of the interchangeable tool which scored 62.80 percentages of satisfaction.

\subsection{Cadaveric experiment trial}

A cadaveric experiment was performed to test the limitation of haptic rendering in Unity when using the high polygon of the haptic model as shown in Figure 12(a) in the real surgical environment. Unity will separate the mesh model to sub-level when the number of vertices is more than 65500 . The plugin will work perfectly with the model which does not subdivide as shown in Figure 12(b), where the distance of each slice is equal to $3 \mathrm{~mm}$ in Z-axis of Unity.

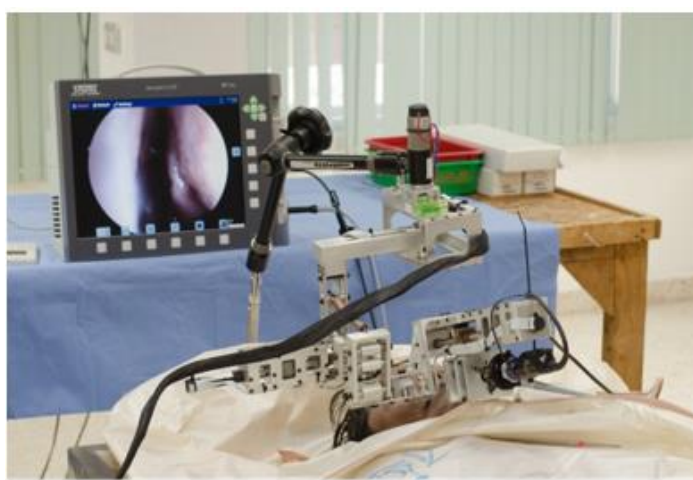

(a)

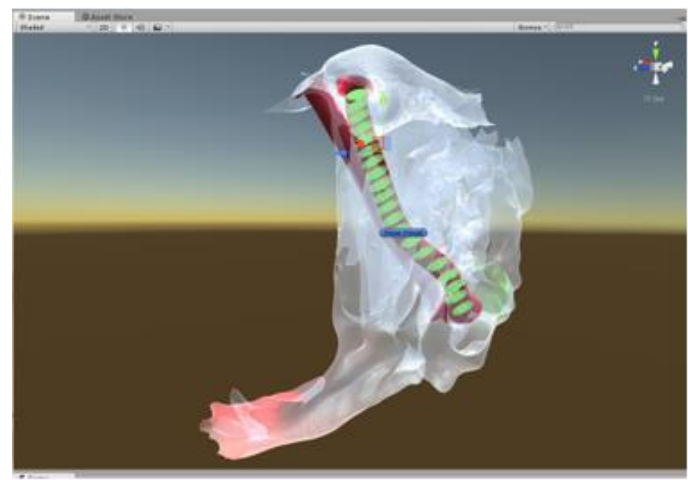

(b)

Figure 12. Cadaveric experiment trial, (a) Experiment setup, (b) GUI pathway cross-section arrangement

\section{CONCLUSION}

In this paper we propose to develop a novel surgical simulator designed for the insertion of the EETS tool based on average pathway model and haptic feedback, starting from the nostril until the area of the sphenoid sinus. The system adheres to the virtual reality (VR) technology for the interaction between the user and the haptic device, while exchanging the force feedback and visual data during the performance of the simulation. As a result, the interaction between the user and the haptic device can increase the experience level by decreasing the time of completion and the error score from the simulator system. Also, the interchangeable tooltip surgical tool test demonstrated that it can perform all the prescribed functions. Finally, it could be validated that the overall system can be used to support the neurosurgeon to overcome the issues they face in an EETS operation. The trend of the data shows that each mode can decrease error score and the time to completion after practicing at least three times. Therefore, the user can learn the motor control 
of hand movement in order to reach the target at the Sphenoid sinus area via the pathway guidance system in the simulator. The pathway guidance system in the simulator can be applied with a robot navigation system to assist the neurosurgeon to handle the surgical tools. This can avoid touching the high-risk area in the nasal cavity and also reduce the time of EETS operation.

\section{ETHICAL APPROVAL}

In this research Cadaveric based experiments were conducted and an Ethical approval (COA. MURA2019/64) statement is enclosed as an electronic supplementary material.

\section{ACKNOWLEDGEMENTS}

This research is financially support by National Research University Funds through Mahidol University and Government Research Budget through Mahidol University (Grant No. 111-2558). Another fund resource by the Computer-Integrated Intelligent Medical System Project under the Thailand National Research University Grant through Mahidol University, Thailand. Authors would like to express their gratitude to BART LAB Researchers Choladawan Moonjaita for her kind technical and intellectual support and also Norrasaet Manourat, Kanin Piemngam as a team to continue this work and organize things together to implement and leading this work at BART LAB.

\section{REFERENCES}

[1] T. Arulampalam, S. Paterson-Brown, A. J. Morris, and M. C. Parker, "Natural Orifice Transluminal Endoscopic Surgery," Annals Royal College of Surgeons of England, vol. 91, no. 6, pp. 456-459, 2009, doi: 10.1308/003588409X464487.

[2] A. Neubauer, "Virtual Endoscopy for Preoperative Planning and Training of Endonasal Transsphenoidal Pituitary Surgery," PhD thesis, VRVis and Vienna University of Technology, pp. 1-152, 2005.

[3] P. Cappabianca, L.M Cavallo, F. Esposito and E. de Divitiis, "Endoscopic endonasal transsphenoidal surgery: procedure, endoscopic equipment and instrumentation," Child's Nervous System, vol. 20, no. 11-12, pp. 796-801, 2004, doi: 10.1007/s00381-004-0933-3.

[4] J. A. Gondim, M. Schops, J. P. C. de Almeida, Lucas Alverne F de Albuquerque, Erika Gomes, Tânia Ferraz et al., "Endoscopic endonasal transsphenoidal surgery: surgical results of 228 pituitary adenomas treated in a pituitary center," Pituitary, vol. 13, no. 1, pp. 68-77, 2010, doi: 10.1007/s11102-009-0195-x

[5] J. D'Haens, K. Van Rompaey, T. Stadnik, et al., "Fully endoscopic transsphenoidal surgery for functioning pituitary adenomas," Surgical Neurology, vol. 72, no. 4, pp 336-340, 2009, doi: 10.1016/j.surneu.2009.04.012.

[6] H. D. Jho and R. L. Carrau, "Endoscopic endonasal transsphenoidal surgery: experience with 50 patients," Journal of Neurosurgery, vol. 87, no. 1, pp. 44-51, 1997, doi: 10.3171/jns.1997.87.1.0044.

[7] D. S. Sethi and K. Kumar, "Endoscopic pituitary surgery," Operative Techniques in Otolaryngology-Head and Neck Surgery, vol. 18, no. 1, pp. 57-64, 2007.

[8] B. M. Pillai, C. Wilasrusmee, and J. Suthakorn, "Observer based dynamic control model for bilaterally controlled MU-LapaRobot: surgical tool force limiting," International Journal of Electrical and Computer Engineering (IJECE), vol. 10, no. 1, pp. 828-839, Feb. 2020, DOI: 10.11591/ijece.v10i1.pp828-839.

[9] S. Nakdhamabhorn, B. M. Pillai, and J. Suthakorn, "Design and development of sensorless based 5-DOF bilaterally controlled surgical manipulator: A prototype," Bulletin of Electrical Engineering and Informatics, vol. 10, no. 2, pp. 619-631, 2021, DOI:10.11591/eei.v10i2.2331

[10] S. S. Wang, L. Xue, J. J. Jing and R. M. Wang, "Virtual reality surgical anatomy of the sphenoid sinus and adjacent structures by the transnasal approach," Journal of Cranio-Maxillofacial Surgery, vol. 40, no. 6, pp. 494-499, 2012, doi: $10.1016 / j . j c m s .2011 .08 .008$.

[11] A. Inoue, T. Ohnishi, S. Kohno et al., "Utility of three-dimensional computed tomography for anatomical assistance in endoscopic endonasal transsphenoidal surgery," Neurosurgical Review, vol. 38, no. 3, pp. 559-565, 2015, doi: 10.1007/s10143-015-0625-3

[12] S. Chumnanvej, B. M. Pillai, and J. Suthakorn, "Surgical robotic technology for developing an endonasal endoscopic transsphenoidal surgery (EETS) robotic system," The Open Neurology Journal, vol. 13, no. 1, pp. 96-106, 2019, doi: 10.2174/1874205X01913010096.

[13] S. Chumnanvej, S. Chalongwongse, B. M. Pillai, and J. Suthakorn, "Pathway and workspace study of endonasal endoscopic transsphenoidal (EET) approach in 80 cadavers," International Journal of Surgery Open, vol. 16, pp. 22-28, 2019, doi: 10.1016/j.ijso.2018.12.002

[14] M. Head and M.J. Head, "Modular Joystick Design for Virtual Reality Surgical Skills Training," MSc Thesis, Mechanical (and Materials) Engineering, University of Nebraska, 2012.

[15] J. J. B. Lim and A. G. Erdman, "A review of mechanism used in laparoscopic surgical instruments," Mechanism and Machine Theory, vol. 38, no. 11, pp. 1133-1147, 2003, doi: 10.1016/S0094-114X(03)00063-6. 
[16] V. Veelen, D. W. Meijer, R. H. Goossens and C. J. Snijders, "New ergonomic design criteria for handles of laparoscopic dissection forceps," Journal of laparoendoscopic \& advanced surgical techniques, Part A., vol. 11, no. 1, pp. 17-26, 2001, doi: 10.1089/10926420150502896.

[17] V. Van Veelen and D. W. Meijer, "Ergonomics and design of laparoscopic instruments: results of a survey among laparoscopic surgeons," Journal of laparoendoscopic \& advanced surgical techniques, Part A., vol. 9, no. 6, pp. 481-489, 1999, doi: 10.1089/lap.1999.9.481.

[18] C. Min Seow, W. Jian Chin, C. Nelson, Akiko Nakamura, Shane M. Farritor, Dmitry Oleynikov, "Articulated Manipulator with Multiple Instruments for Natural Orifice Transluminal Endoscopic Surgery," Journal of Medical Devices, vol. 7, no. 4, pp. 1-10, 2013, doi: 10.1115/1.4025183.

[19] T. R. Coles, D. Meglan and N. W. John, "The Role of Haptics in Medical Training Simulators: A Survey of the State of the Art," IEEE Transactions on Haptics, vol. 4, no. 1, pp. 51-66, 2011, doi: 10.1109/TOH.2010.19.

[20] T. Coles and N. John, "Integrating haptics with augmented reality in a femoral palpation and needle insertion training simulation," IEEE Transactions on Haptics,, vol. 4, no. 1, pp. 199-209, 2011, doi: 10.1109/TOH.2011.32.

[21] A. Pößneck, E. Nowatius, C. Trantakis, H.Cakmak, H. Maass, U. Kühnapfel et al., "A virtual training system in endoscopic sinus surgery," International Congress Series, vol. 1281, pp. 527-530, 2005, doi: 10.1016/j.ics.2005.03.184.

[22] G. Rosseau, J. Bailes, R. Del Maestro, Anne Cabral, Nusrat Choudhury, Olivier Comas, Patricia Debergue et al., "The Development of a Virtual Simulator for Training Neurosurgeons to Perform and Perfect Endoscopic Endonasal Transsphenoidal Surgery," Neurosurgery, vol. 73, no. 4, pp. S85-S93, 2013, doi: 10.1227/NEU.0000000000000112.

[23] G. J. Wiet, D. Stredney and D. Wan, "Training and simulation in otolaryngology," Otolaryngologic Clinics of North America, vol. 44, no. 6, pp. 1333-1350, 2011, doi: 10.1016/j.otc.2011.08.009.

[24] S. P. Dakua, J. Abinahed, A. Zakaria, Shidin Balakrishnan, Georges Younes, Nikhil Navkar et al., "Moving object tracking in clinical scenarios: application to cardiac surgery and cerebral aneurysm clipping," International Journal for Computer Assisted Radiology and Surgery, vol. 14, no. 12, pp. 2165-2176, 2019, doi: 10.1007/s11548-01902030-z.

[25] P. Strakos, M. Jaros, T. Karasek, T. Kozubek, P. Vavra, T. Jonszta, "Review of the Software Used for 3D Volumetric Reconstruction of the Liver," International Journal of Computer and Information Engineering, vol. 9, no. 2, pp. 422-426, 2015, doi: 10.5281/zenodo.1099182.

[26] J. Egger, T. Kapur, C. Nimsky and R. Kikinis. "Pituitary Adenoma Volumetry with 3D Slicer," PLoS One, vol. 7, no. 12, pp. 1-7, 2012, doi: 10.1371/journal.pone.0051788.

[27] N. S. Hasan, N. Rosmin, S. A. Khalid, Dygku. A. Awg. Osman, Baharuddin Ishak, A. H. Mustaamal, "Harmonic suppression of shunt hybrid filter using LQR-PSO based," International Journal of Electrical and Computer Engineering (IJECE), vol. 7, no. 2, pp. 869-876, 2017, doi: 10.11591/ijece.v7i2.pp869-876.

[28] S. A. Ali, M. F. Miskon, A. Z. Hj Shukor, Marwan Qaid Mohammed, "The Effect of Parameters Variation on Bilateral Controller," International Journal of Power Electronics and Drive System (IJPEDS), vol. 9, no. 2, pp. 648-659, 2018, doi: 10.11591/ijpeds.v9n2.pp648-659.

[29] B. M. Pillai and J. Suthakorn, "Motion control applications: observer based DC motor parameters estimation for novices," International Journal of Power Electronics and Drive System (IJPEDS), vol. 10, no. 1, pp. 195-201, 2019, doi: 10.11591/ijpeds.v10.i1.pp195-210. 